\section{ORIGINAL RESEARCH}

B. Borg

M.T. Modic

N. Obuchowski

G. Cheah

\title{
Pedicle Marrow Signal Hyperintensity on Short Tau Inversion Recovery- and T2-Weighted Images: Prevalence and Relationship to Clinical Symptoms
}

BACKGROUND AND PURPOSE: Lumbar pedicle marrow hyperintense signal on T2- and STIR-weighted images is not an uncommon finding. We hypothesize that these marrow signal intensity changes and their behavior within the pedicle are associated with clinical symptoms and that their improvement or resolution correlates with clinical improvement. We investigated the prevalence of these pedicle marrow changes, associated morphologic abnormalities, and the relationship to symptoms over time.

MATERIALS AND METHODS: Prevalence was evaluated prospectively in 246 patients and retrospectively in 400 patients. To analyze the relationship between changes in signal intensity over time and symptoms, a third group of 30 patients was followed clinically for assessment of pain and functional limitation and with MR studies for 18 months or until signal intensity changes resolved.

RESULTS: The prevalence of pedicle marrow hyperintensity on T2 and STIR-weighted sequences was $1.7 \%$. Associated morphologic abnormalities were pars interarticularis and pedicle fractures and degenerative facets. In the longitudinal study, pedicle marrow signal hyperintensity on T2- and STIR-weighted images resolved in 17 patients and persisted in 5 patients. The extent, intensity, and resolution of signal intensity changes significantly related to the degree of functional limitation $(P=$ .01).

CONCLUSIONS: Resolution of pedicle marrow hyperintensity on T2 and STIR-weighted images was associated with improved functional testing and a trend toward decreased pain.

ABBREVIATIONS: $\mathrm{Cl}=$ confidence interval; $\mathrm{DFC}=$ degenerative facet change; Pars = pars interarticularis fracture; $\mathrm{Ped}=$ pedicle fracture; STIR $=$ short tau inversion recovery

C hanges in MR signal intensity within the pedicles of the lumbar vertebrae are not uncommon and have been reported in association with spondylolysis and degenerative facet changes. ${ }^{1-5}$ In these reports, the signal intensity changes were categorized in a manner similar to vertebral body endplate marrow changes associated with degenerative disk disease that were described by Modic. ${ }^{6}$ Although these changes in the MR signal intensity take 3 main forms (types 1-3), of primary interest here are the type 1 pedicle marrow signal intensity changes. Typically, type 1 changes are defined as decreased signal intensity on T1 imaging and increased signal intensity on T2/STIR sequences. Type 2 marrow changes are defined as increased signal intensity on T1 images and more subtle increased signal intensity on T2. Type 1 pedicle marrow signal intensity changes are more strongly associated with low back pain and less stable lesions than types 2 and $3 .^{7}$ For purposes of brevity, we henceforth refer to pedicle marrow signal hyperintensity on T2 and STIR-weighted images as type 1 pedicle marrow signal intensity changes.

These type 1 pedicle marrow signal intensity changes and their resolution in some patients suggest a healing or stabiliz-

Received September 14, 2010; accepted after revision January 25, 2011.

From the Imaging (B.B., G.C.) and Neurological (M.T.M.) Institutes, and Quantitative Health Sciences (N.O.), Cleveland Clinic, Cleveland, Ohio.

Please address correspondence to Michael T. Modic, MD, FACR, Cleveland Clinic, 9500 Euclid Ave, Cleveland OH 44195; e-mail: modicm1@ccf.org

Indicates article with supplemental on-line table.

http://dx.doi.org/10.3174/ajnr.A2588 ing process; so, understanding their behavior and clinical correlates may have implications for the prognosis and management of the underlying etiology. Thus, we investigated the prevalence of type 1 pedicle marrow signal intensity changes, identified the associated morphologic abnormalities, and examined the behavior of the changes and how they were related to patient symptoms over time. We hypothesized that these type 1 pedicle marrow signal intensity changes and their behavior within the pedicle are associated with clinical symptoms and may be similar to and behave like type 1 pedicle marrow signal intensity changes in the vertebral body.

\section{Materials and Methods}

This study received approval from our institutional review board and complied with Health Insurance Portability and Accountability Act regulations. All patients whose data were collected prospectively provided signed informed consent.

\section{Prevalence of Type 1 Pedicle Marrow Signal Intensity Changes}

We studied 2 distinct populations, 1 prospective population and 1 retrospective population. The prospective group consisted of $246 \mathrm{pa}-$ tients with acute low back pain, radiculopathy, or both, who presented within 3 weeks of pain onset and were recruited for an ongoing prospective study. ${ }^{8}$ The patients were imaged on 1.5T MR scanners (Symphony; Siemens, Erlangen, Germany). T1 sagittal images (TR/ TE: 500/12; matrix $192 \times 256$; 3 averages; sequence time 4 minutes, 20 seconds); T1 axial images (TR/TE: 600/12; matrix $192 \times 256$; 3 averages; sequence time 4 minutes, 40 seconds); and T2 sagittal and axial 
fast spin-echo images (TR/TE: 5000/120; matrix $192 \times 256$; 3 averages; sequence time 4 minutes, 42 seconds.

The second group was retrospectively identified from patient records. Records were selected if the patient had undergone MR imaging for low back pain at our institution and had neither trauma nor malignancy as the primary clinical concern or as a finding on the examination. The last 50 male and 50 female patients with MR imaging for low back pain from January 1, 2004, to December 31, 2004, were identified in each of 4 age ranges $-<30,30-49,50-59$, and $\geq 60$ years of age-for 400 patients in total. All patients were imaged on 1.5T MR scanner systems (Siemens). In addition to the above-mentioned protocol, each patient received STIR sagittal imaging (TR/TE: 4200/60; matrix $192 \times 256$; 3 averages; sequence time 4 minutes, 38 seconds). Images were reviewed by 2 neuroradiologists for consensus interpretation regarding the presence of type 1 pedicle marrow signal intensity changes. The diagnoses underlying these changes were recorded for both groups.

\section{Presumed Etiology and Longitudinal Changes in Type 1 Signal Intensity}

To investigate changes in signal intensity over time and their relationship to symptoms, we identified a third group of patients with type 1 pedicle marrow signal intensity changes during routine clinical evaluation for low back pain from December 2004 to March 2007. These patients were entered into a data base to catalog morphologic abnormalities associated with the marrow changes. We then recruited patients from this data base. To be included, patient care must have been documented in our institution's electronic health record (Epic Care; Epic Systems, Verona, Wisconsin) and had sufficient clinical documentation (including Roland and Likert pain severity scale scores obtained by phone interview within 1 week of the imaging follow-up) to determine the clinical course and had at least 1 follow-up MR examination at our institution. Patients were followed for at least 18 months or until pedicle marrow signal intensity changes resolved, whichever occurred first; the 18-month stopping point was arbitrarily chosen. For all patients in the data base, images were reviewed by 2 neuroradiologists for concordant interpretations of the presence or absence of type 1 pedicle marrow signal intensity and distribution of signal intensity changes. All images also were reviewed for changes in MR signal intensity over time, and this change was quantified. Readers were blinded to patient identity and clinical data. Ancillary morphologic findings also were recorded.

To provide semiquantification for purposes of assessing longitudinal change, type 1 pedicle marrow signal intensity change was quantified as follows: the signal hyperintensity was graded by dividing the pedicle, facets, and pars interarticularis into 7 zones (roughly, 4 quadrants of the pedicle on a sagittal STIR image, plus the superior facets, inferior facets, and pars interarticularis) and summing the zones of involvement. The marrow edema signal intensity was quantified by placing a circular region of interest on the area of maximal pedicle hyperintensity and then determining the intensity score according to a 4-point linear scale defined by the intensity of normal vertebral body marrow (0, lowest intensity) and CSF (3, highest intensity) on that axis.

\section{Statistical Analysis}

For the prevalence of type 1 pedicle marrow signal intensity changes, 95\% CIs were constructed for both patient populations, by using the normal distribution approximation. Fisher exact test was used to test
Table 1: Structural abnormality compared by age for 91 data base patients with low back pain and type 1 pedicle marrow changes

\begin{tabular}{lccc}
\hline & Age $<30 \mathrm{yr}$ & Age $30-50 \mathrm{yr}$ & Age $\geq 50 \mathrm{yr}$ \\
\hline No. with type 1 changes & 27 & 17 & 47 \\
Sex (M/F) & $24 / 3$ & $3 / 14$ & $16 / 31$ \\
Diagnosis & & & \\
Fracture, pars & 17 & 1 & 5 \\
Fracture, pedicles & 5 & 1 & 1 \\
Degenerative facet disease & 0 & 15 & 41 \\
No definitive diagnosis & 5 & & \\
\hline
\end{tabular}

the association between the presence of type 1 pedicle marrow signal intensity changes and patient characteristics.

For the longitudinal study, Wilcoxon 2-sample tests were used to test whether patient age, baseline pain, and baseline signal intensity scores differed between patients with signal intensity resolution and those without resolution within 18 months. Kendall tau correlation coefficients were used to assess the correlation between patient outcomes (pain score, Roland score, and change in pain score from baseline) and pedicle marrow signal intensity (intensity, distribution, and change from baseline in intensity and distribution). Significance was set at .05. Because these investigations were exploratory, no power calculations were performed.

\section{Results}

\section{Prevalence of Type 1 Pedicle Changes}

In the prospective group, the prevalence of type 1 pedicle marrow signal intensity change was $1.6 \%(4 / 246 ; 95 \% \mathrm{CI}, 0.0 \%$ $3.2 \%)$. These patients were female $(P=.14)$, with a mean age of 54 years (range, $48-60$ years). Three patients had low back pain, and the fourth had radicular symptoms. In each case, the marrow changes were associated with advanced degenerative changes in the adjacent facet joints.

In the retrospective group (with STIR sequence), the prevalence was $1.8 \%$ (7/400; 95\% CI, $0.0 \%-3.0 \%)$. Two patients were younger than 30 years, both male. No additional abnormalities were seen. One patient was in the 30-49 year age group and 1 patient was in the $50-59$ year group; both patients were female, with moderate or advanced degenerative changes in adjacent facet joints. Three patients ( 2 male, 1 female) were in the $\geq 60$ age group, all with moderate or advanced degenerative changes in adjacent facet joints.

Type 1 pedicle marrow signal intensity changes were not significantly associated with either age or sex in the retrospective group. The conspicuity of the signal intensity changes was greater on STIR than T2-weighted sequences but a direct comparative accuracy between the 2 sequences was not performed.

\section{Etiology and Longitudinal Changes}

At the time of this study, our data base contained records for 91 patients. Age distribution and diagnoses are listed in Table 1. Thirty patients met the inclusion criteria for longitudinal investigation.

Of the 30 patients analyzed longitudinally, the average duration of follow-up for all patients was 12 months (range, 4-22 months). Eight did not complete the study because they were lost to or declined additional follow-up. The remaining 22 patients were followed for at least 18 months or until the mar- 
row changes resolved, whichever occurred first. The type 1 pedicle marrow signal intensity resolved in 17 patients (mean age, 39 years; mean time to resolution, 11 months; range, 4-22 months) and persisted in 5 patients. On-line Table 1 summarizes the clinical course and signal intensity changes in the 30 patients.

There were no statistically significant differences in age, baseline pain score ( 8.0 versus 5.8 ), baseline signal intensity (4.3 versus 5.0 ), or baseline signal intensity distribution (8.6 versus 9.6) for patients whose pedicle marrow signal intensity resolved compared with patients whose pedicle marrow signal intensity did not resolve.

Roland function score modestly correlated with signal intensity $(r=0.28, P=.01)$, signal intensity distribution $(r=$ $0.25, P=.02)$, and change in distribution since baseline $(r=$ $0.29, P=.01$ ). Higher intensity and distribution scores were associated with higher (worse) Roland scores, and greater reductions in distribution scores from baseline were associated with lower (better) Roland scores. The mean pain score increased slightly in association with higher pedicle marrow intensity and distribution scores. In contrast, the mean Roland score increased markedly with higher pedicle marrow intensity and distribution scores.

The mean pain scores for those with resolution were 8.0 at baseline and 5.3 at last follow-up. In the 7 patients without measurable improvement in pain scores, 5 had degenerative facet changes. Self-reported pain scores tended to improve over time with concordant resolution of marrow signal intensity changes, but this was not statistically significant.

Of the 5 patients without signal intensity resolution by 18 months, 3 had an improvement in the distribution and signal intensity and 2 remained unchanged. The mean pain scores were 5.8 at baseline and 4.2 at 18 months.

The 8 patients who were lost to follow-up were followed for an average of 9 months. At last follow-up examination, the signal intensity had improved in 4 , remained the same in 3 , and worsened in 1 . None had complete resolution of the type 1 pedicle marrow signal intensity changes. The mean pain scores were 7.0 at baseline and 3.9 at last follow-up. In 5 of the 8 patients, the pain score had improved at the last follow-up study.

In 6 of the 17 patients with resolution, subsequent conversion to type 2 marrow changes was seen on the follow-up studies (On-line Table 1).

In addition to pedicle signal intensity change, 9 of 30 patients had increased signal intensity in the soft tissues adjacent to the facet joints and posterior lateral elements, on STIRweighted sequences. Four patients had spondylolysis, 3 had degenerative facet changes, and 2 had a pedicle fracture. The soft tissue changes resolved within the follow-up period in all 9 patients. In 6 of 9 patients, symptoms improved or resolved.

\section{Discussion}

We found type 1 pedicle marrow signal intensity changes in $1.7 \%$ of patients who underwent MR of the lumbar spine for back pain. These were most commonly associated with 3 distinct structural abnormalities: degenerative facet disease (Fig 1) and fractures of the pars interarticularis (Fig 2) or pedicle (Figs 3 and 4). Although pars and pedicle fractures were more common in younger patients and degenerative facet disease
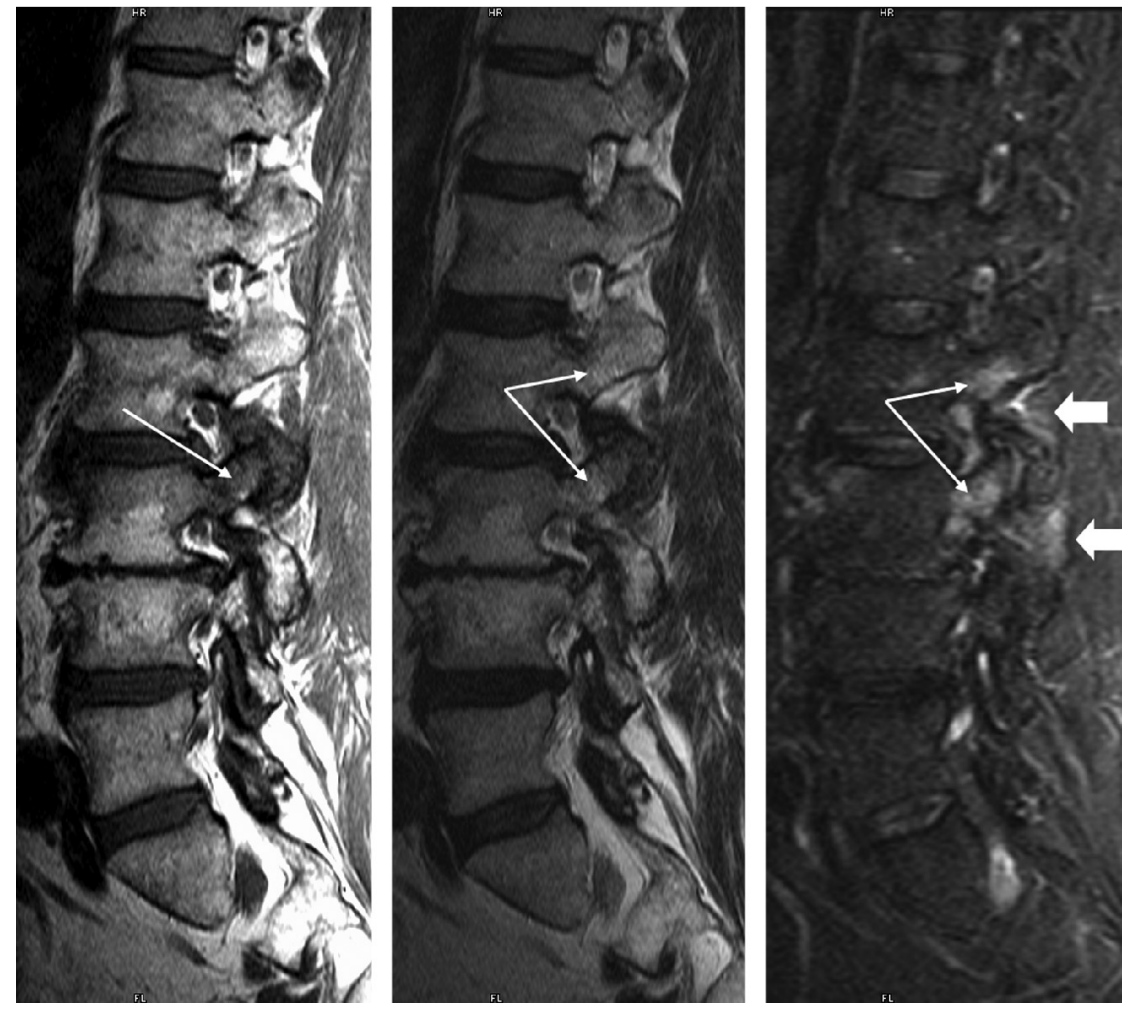

Fig 1. Type 1 pedicle marrow signal intensity changes associated with degenerative facet disease. A 60-year-old patient with back pain and degenerative facet changes. Parasagittal T1, T2, and STIR images are shown, left to right. On the T1 image, hypointensity is present within the pedicle and superior articular facet of L3 (/ong arrows). Hyperintensity is noted within this same region on T2- and STIR-weighted sequences (long arrows) as well as within the pedicle of L2. Note the subtle hyperintensity within the soft tissues adjacent to the facet joints at both levels on the STIR image (block arrows). 

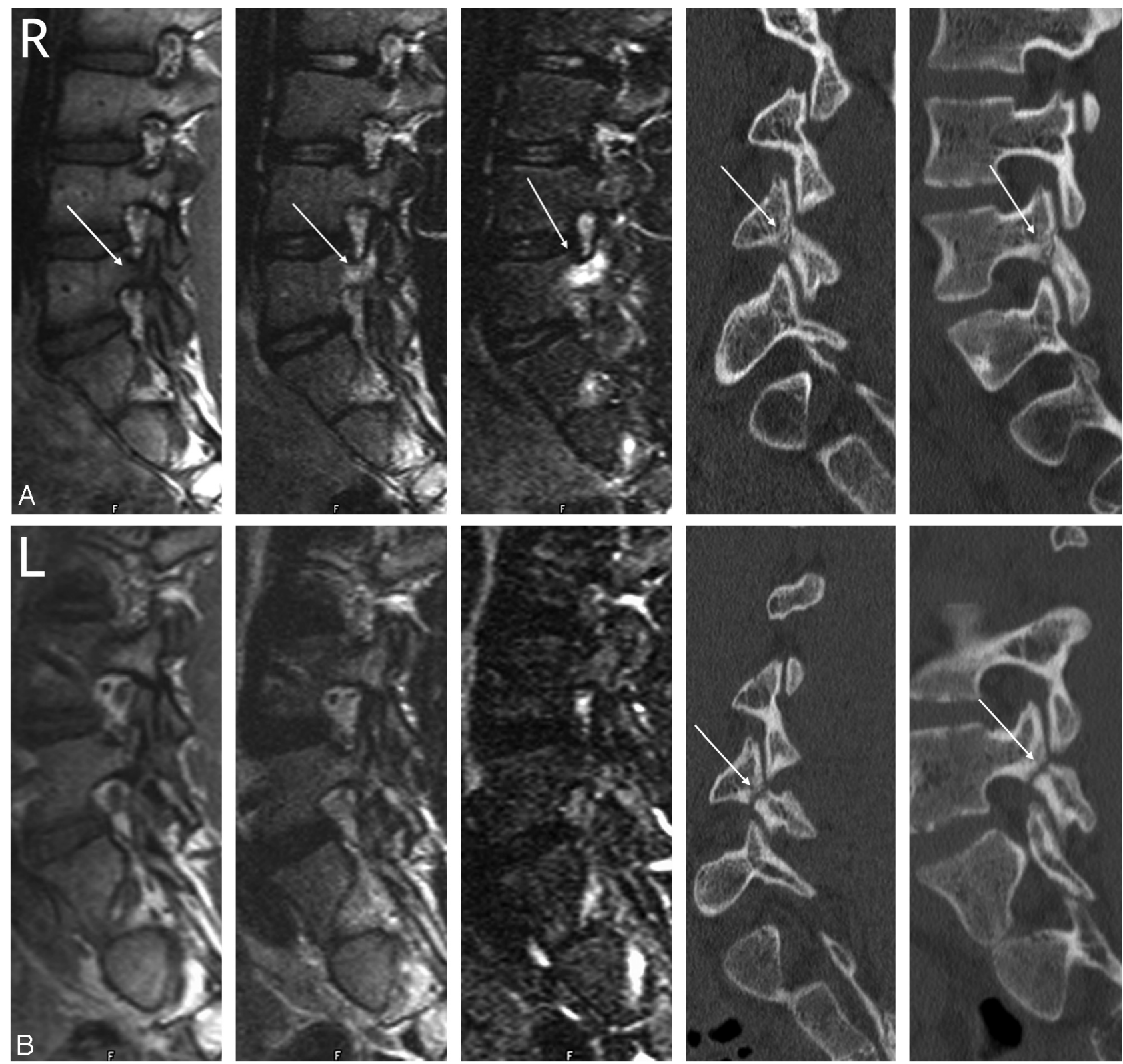

Fig 2. Type 1 pedicle marrow signal intensity changes associated with an early to progressive pars fracture and absence of signal intensity changes in a terminal spondylolysis in an 18-year-old man with back pain and bilateral spondylolysis. Right $(A)$ and left $(B)$ parasagittal MR images of the lumbar spine. In each figure, the T1, T2, STIR, and multiplanar reformatted oblique and parasagittal CT images are shown, from left to right. A, Hypointensity of the L4 right pedicle is evident on T1 with increased signal intensity on T2- and STIR-weighted images (arrows). A relatively acute fracture is noted through the pars interarticularis on the CT images (arrow). B. Signal intensity of the left pedicle is normal on all sequences. The CT images reveal an older pars fracture (arrows).

more so in older patients, neither were exclusive to a single age group. Although much less common and not addressed in this study, similar hyperintense pedicle marrow signal intensity on T2- and STIR-weighted images may be associated with infection, hemangioma, osteoid osteoma, or other bony neoplasms. We determined that both the extent and intensity of these marrow changes significantly related to the degree of functional limitation that was due to low back pain. Self-reported pain scores tended to improve over time and with concordant resolution of marrow signal intensity changes, but this was not statistically significant.

Type 1 pedicle marrow signal intensity changes have been best characterized in the pediatric patient with low back pain associated with fractures of the pars interarticularis.
Studying pedicle signal intensity changes on MR in pediatric patients $(n=37)$, Sairyo ${ }^{9}$ concluded that type 1 pedicle marrow signal intensity changes may reflect active stress changes in the underlying bone, a hypothesis others have proposed. ${ }^{1-3}$ There is a large amount of literature on a similar marrow signal intensity pattern in joints outside the spine where symptoms and abnormal marrow signal intensity in long bones correlate with clinical symptoms and trauma or response to stress. ${ }^{10-14}$

Likewise, the association of type 1 pedicle marrow signal intensity changes with pars and pedicle fractures and degenerative facet changes suggests they are related to biomechanical stress created by abnormal spinal segment motion. This or a similar mechanism may be at play in the vertebral bodies 


\section{Left}

02/09/05
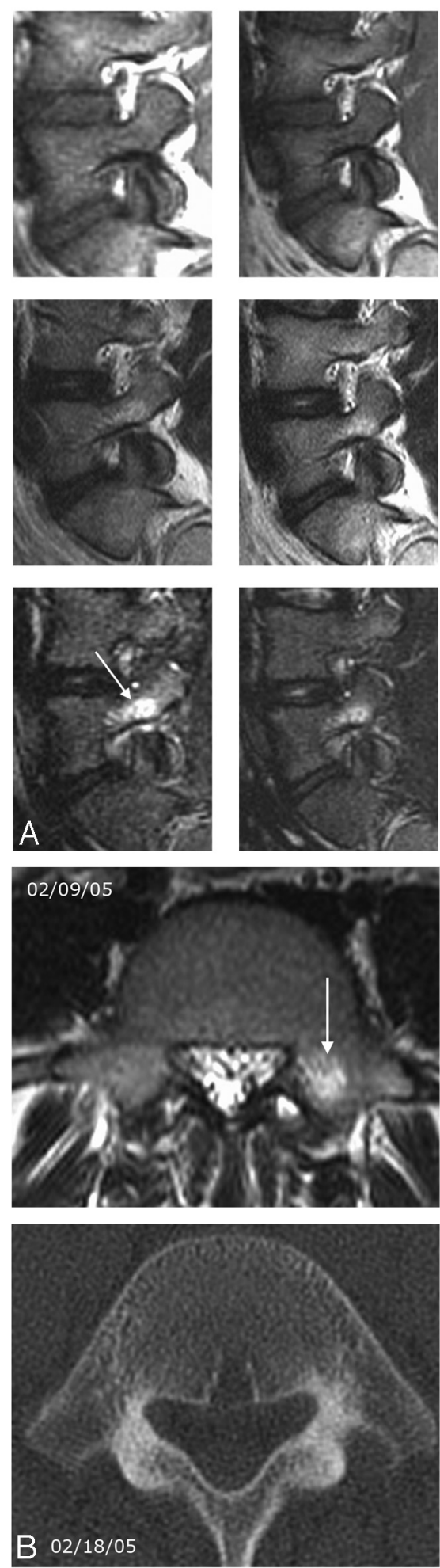

08/04/05
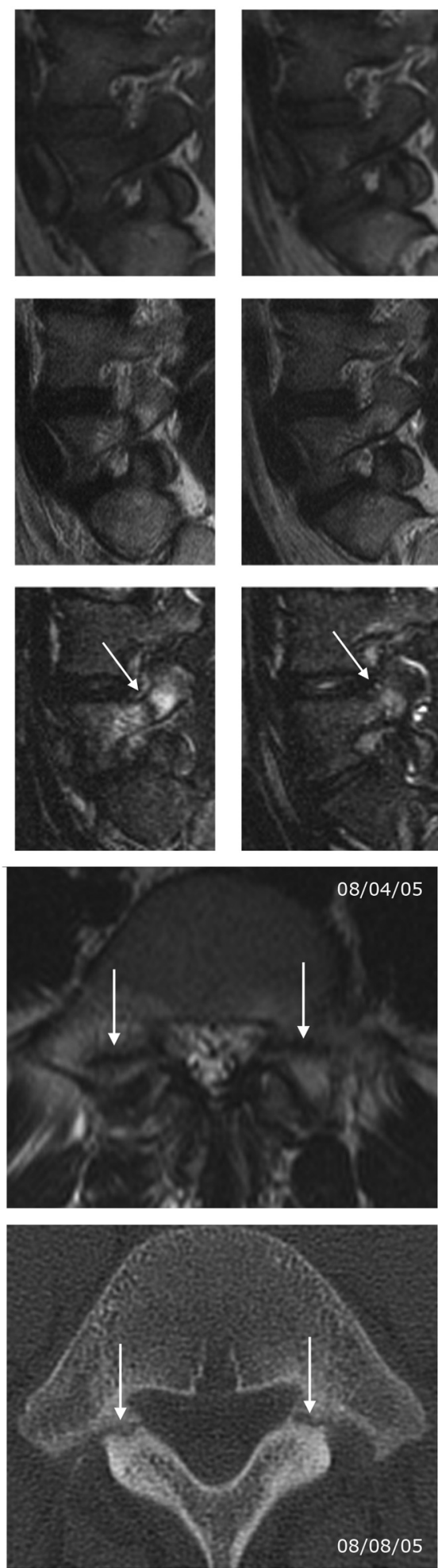

Fig 3. Type 1 pedicle marrow signal intensity changes associated with evolving pedicle fractures in a 13-year-old male adolescent. $A$, Left type 1 pedicle marrow signal intensity changes, which are best appreciated on the STIR sequence. From top to bottom, each row shows T1-, T2-, and STIR-weighted sequences. The MR study on February 9, 2005, demonstrates increased signal intensity within the left pedicle and proximal superior facet, which is best appreciated on the STIR sequence (arrow). He was treated conservatively and the back pain improved. The follow-up study on March 23, 2005, demonstrates some improvement in the hyperintensity of the left L5 pedicle. A follow-up MR study on August 4, 2005, demonstrates increased type 1 pedicle marrow changes of $\mathrm{L} 5$ bilaterally with a fracture line at the junction of the pedicle and superior articular facets (arrow). A follow-up study from February 20, 2006, shows some improvement of the increased signal intensity on STIR and T2 (arrows), and the previous fracture line is no longer appreciated. Similar changes were seen on the right. The patient's back pain was less severe but still present. B. Axial T2 MR images (top row) through the L5 pedicle on February 9, 2005, and August 4, 2005, and axial CT images (bottom row) through the L5 pedicle on February 18, 2005, and August 8, 2005. The CT obtained on February 18, 2005, was read as negative for fracture. The follow-up MR image demonstrates bilateral fracture lines through the L5 pedicles. The follow-up CT from August 8, 2005, shows clearly demarcated fractures through the junction of the pedicle and superior facet of L5 bilaterally (arrows). 

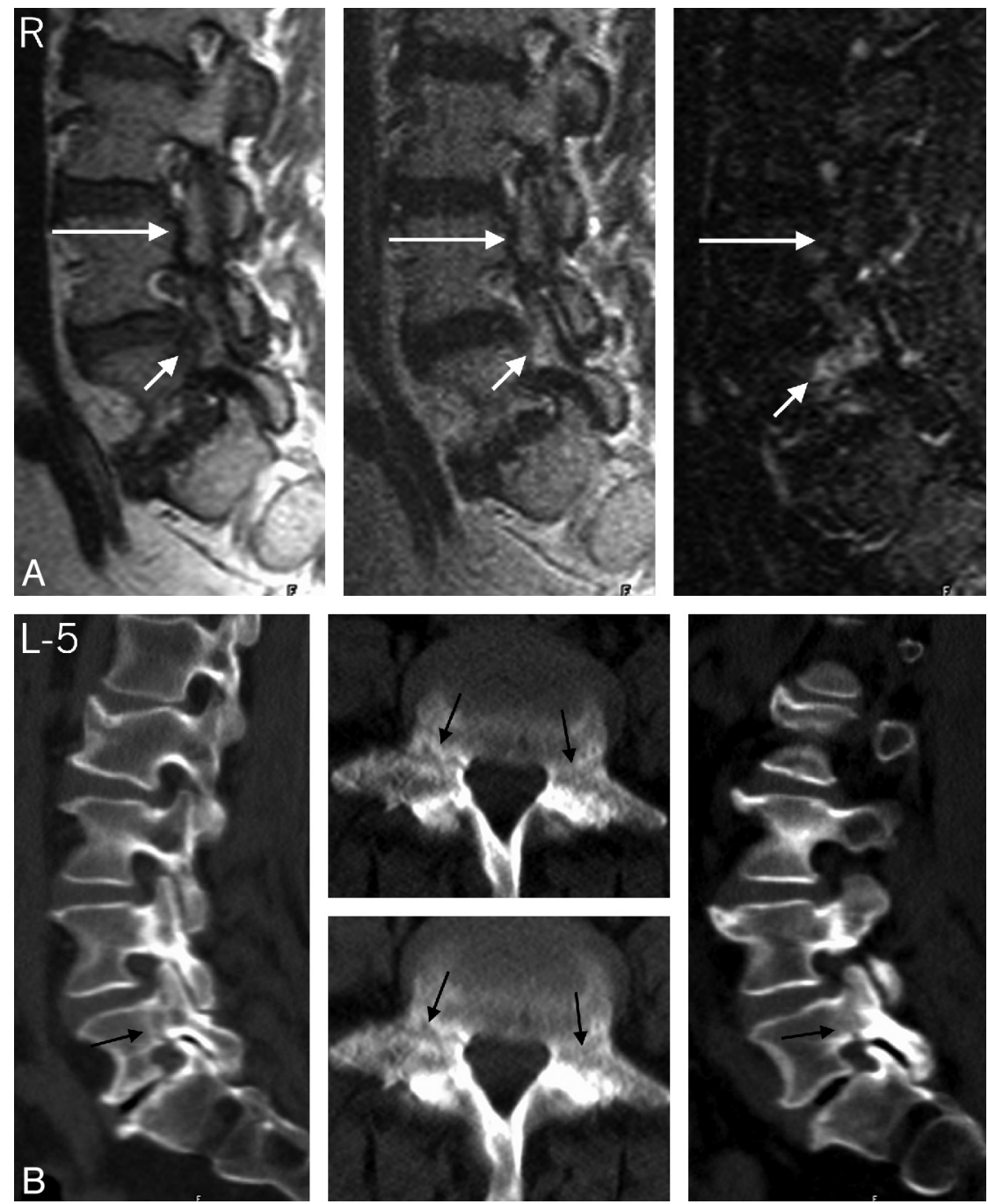

Fig 4. Type 1 pedicle marrow signal intensity changes associated with new and old pedicle fractures in a 64-year-old woman. A, Right parasagittal T1-, T2-, and STIR-weighted images that demonstrate a discrete fracture line through the pedicles of $L 4$ bilaterally without pedicle marrow signal intensity changes (long arrow) and a less obvious fracture line on $\mathrm{T} 1$ images through the L5 pedicle with concomitant type 1 pedicle marrow changes (short arrows). Signal intensity changes at L5 and clinical symptoms had resolved at 8 months of follow-up. $B$, Right and left parasagittal multiplanar reformatted CT images through the lumbar spine and axial images through the pedicles of L5. Note the lucent appearance of the subacute fracture site through the pedicles on the parasagittal multiplanar reformatted images and adjacent axial CTs and the less well-defined fracture and adjacent marrow signal intensity alteration through the pedicles on the axial MR images (arrows).The signal intensity change is more obvious on the axial MR image on the right. Note the sclerotic appearance of an older fracture site through the $L 4$ pedicle on the CT images.
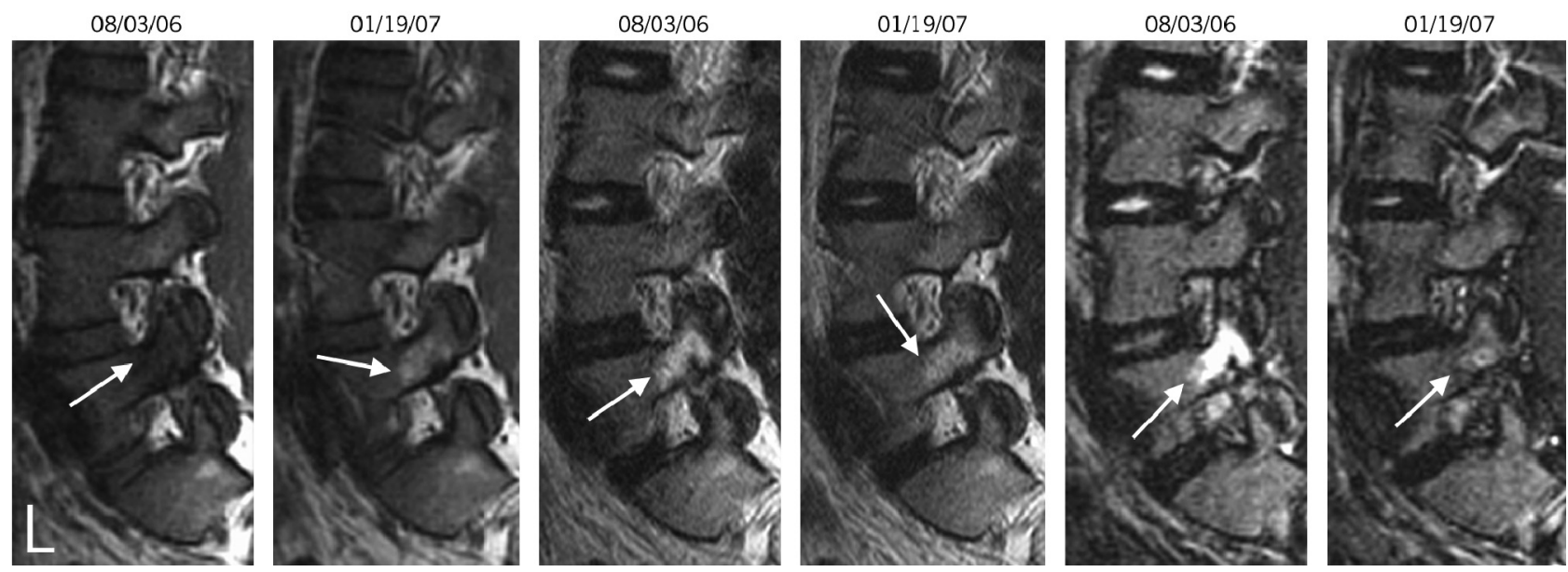

Fig 5. A 25-year-old man with back pain. The left parasagittal T1, T2, and STIR images (left to right) from August 3, 2006, and January 19, 2007, which demonstrate type 1 pedicle marrow changes on the August 3, 2006, study. The hyperintensity on T2 and STIR images has resolved by the second study. On the T1 images, the decreased marrow signal intensity (type I) noted initially (August 3, 2006) has converted to a type 2 marrow (increased signal intensity on T1) on the second study (January 19, 2007). 

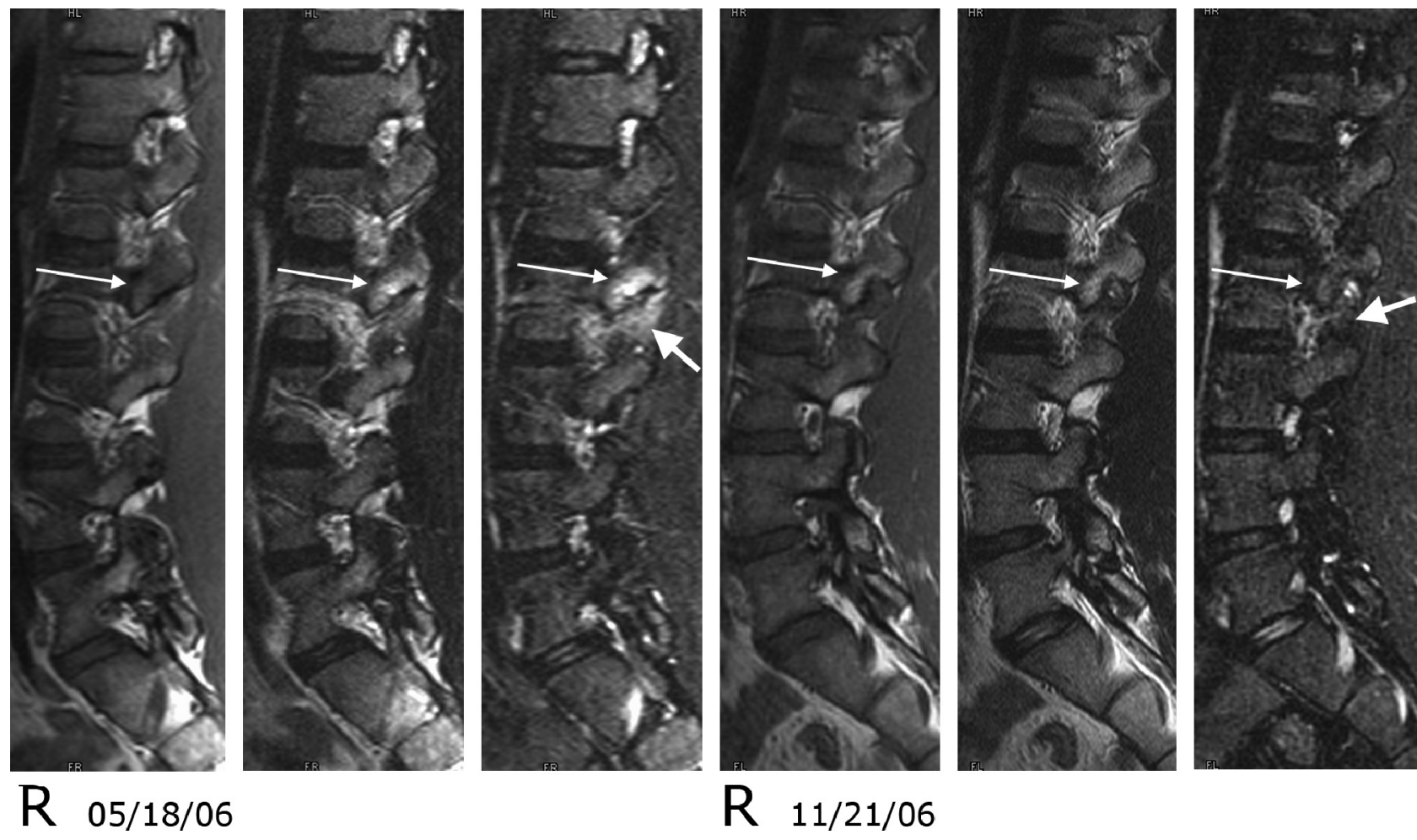

Fig 6. Type 1 pedicle marrow signal intensity changes and associated soft tissue hyperintensity on T2- and STIR-weighted sequences associated with pars fractures of L2 in a 14-year-old female adolescent. Right parasagittal T1, T2, and STIR images from May 18, 2006, and November 21, 2006. On the initial study, type 1 pedicle marrow signal intensity changes are noted within the L2 pedicle and superior articular facet (long arrow). The signal intensity also is increased within the soft tissue adjacent to the L2 superior facet (short arrow) on the STIR-weighted sequences. On the follow-up study, the marrow changes have converted from type 1 to type 2 (long arrow), and the soft tissue changes have resolved (short arrow). The patient was improved clinically.

adjacent to degenerated disks. Type 1 vertebral body marrow changes are associated with degenerative disk disease and more fluid and variable than type $2^{6,7,15-17}$ Type 1 pedicle marrow signal intensity changes seem to be associated with a higher prevalence of active low back symptoms ${ }^{15,16,18-20}$ than types 2 and 3 and have a reported prevalence of 4\%-15\% in patients with low back pain. Type 1 endplate marrow changes also have been noted to develop in $8 \%$ of patients after diskectomy $^{21}$ and in $40 \%$ of patients after chemonucleolysis, ${ }^{22}$ both of which may be viewed as models of accelerated disk degeneration associated with altered biomechanical stress, be it loading, motion, or a combination. That these changes may reflect altered biomechanical stress is further supported by the observation that type 1 changes can convert to type 2 or a more normal-appearing marrow and that conversion is accelerated and possibly facilitated by fusion and instrumented stabilization. ${ }^{17,23-26}$ Six of our 16 patients with signal intensity resolution underwent conversion to a type 2 marrow change (Fig 5).

When stressed, bone behaves according to the Wolff law. ${ }^{27}$ When bone is consistently stressed, it may develop microfractures followed by osteoblastic repair activity. These microfractures demonstrate abnormal radionuclide uptake during scintigraphy. MR signal intensity changes may reflect biomechanical stresses that result in remodeled trabecular bone with microfractures and associated marrow changes. ${ }^{13,28,29}$ Others have postulated that a continuum in the physiologic response to stress fractures represents an imbalance or uncoupling between bone resorption and bone formation. ${ }^{30-32}$ Subsequent complete fractures are probably a result of exceeding trabecular and cortical limits to withstand the forces applied.

In pedicle fractures, stress changes communicated through the pedicle probably produce the signal hyperintensity on STIR and the fracture itself. In the case of pars interarticularis fractures, the pedicle changes are probably the result of altered biomechanical stress that manifests in the pedicle due to altered spinal segment forces secondary to the pars fracture. Likewise, pedicle changes in degenerative facet disease are probably secondary stress changes. Concomitant CT and MR examinations were not part of this study, but in the 2 patients who did have CT and MR, signal intensity changes preceded the development of obvious bony fractures (Fig 3). Although speculative, it may be that the resolution of the marrow changes and improved functional and pain levels are associated with healing, either bony or fibrous, and reduced biomechanical stress. We do not know whether or why the changes might resolve in patients without demonstrable fractures, but we hypothesize that the resolution might be related to remodeled bone that has greater stability or healed microfractures. The resolution of changes in patients who have degenerative facets remains the most perplexing issue because this is a chronic process, unless some degree of unidentified stabilization does occur.

Posterior paraspinal soft tissue hyperintensity that is more conspicuous on STIR sequences has been reported in association with degenerative spinal changes. ${ }^{33,34}$ Adjacent soft tissue hyperintensity on STIR sequences in almost a third of our patients suggests that in the symptomatic stage, altered biome- 
chanical forces affect not just the bone but exert secondary effects within the adjacent soft tissues as well. We saw these soft tissue changes in patients with pars and pedicle fractures as well as degenerative facet disease. Their resolution along with the marrow hyperintensity on STIR would support an acute stage that alters biomechanics and is then followed by subsequent stabilization and adaptation over time (Figs 1 and 6).

Further questions are whether these changes indicate a higher risk for fracture and whether different therapies can alter the clinical course, by using marrow changes as a marker. In a study by Sakai et a ${ }^{35}$ of 17 adolescent patients with fresh spondylolysis, signal intensity changes resolved by 3 months in most patients but persisted longer in patients who did not comply with conservative treatment. Our observations would benefit from further, systematic evaluation in a larger patient population with more rigorous follow-up and concordant CT examinations, which could provide important information relative to boney trabecular and cortical changes. Finally, such a study should control for different types of clinical intervention versus symptom and pedicle marrow signal intensity resolution that was not done in this study.

\section{Conclusions}

Our results suggest that type 1 pedicle marrow signal intensity is a marker for a spectrum of bony changes (eg, remodeling, stress fracture, and complete fracture) most commonly associated with 3 distinct structural abnormalities (pedicle fractures, pars fractures, and degenerative facets) and clinically observable pain and functional limitation. The resolution of these changes is associated with improved functional testing and a trend toward decreased pain. If a component of this process is altered biomechanical force, recognizing these changes at an early stage may permit modification of therapy for a subset of symptomatic patients with type 1 pedicle marrow signal intensity changes. Furthermore, in adolescents, where the question of a pars or pedicle fracture is often an important differential consideration in the patient with back pain, MR imaging should play a more routine role in the evaluation. Type 1 pedicle marrow signal intensity changes may be important in identifying a subgroup that is at risk for fracture or who may benefit from more conservative measures to reduce biomechanical stress.

Disclosures: Nancy Obuchowski, Research Support (including provision of equipment or materials): Siemens Medical, Inc.; iCAD; Details: I am a biostatistician. My department contracts with Siemens and iCAD for data analysis. I perform some of the data analysis, but I personally receive nothing from Siemens or ICAD.

\section{References}

1. Ulmer JL, Elster AD, Mathews VP, et al. Lumbar spondylolysis: reactive marrow changes seen in adjacent pedicles on MR images. AJR Am J Roentgenol 1995;164:429-33

2. Ulmer JL, Mathews VP, Elster AD, et al. MR imaging of lumbar spondylolysis: the importance of ancillary observations. AJR Am J Roentgenol 1997; 169:233-39

3. Hollenberg GM, Beattie PF, Meyers SP, et al. Stress reactions of the lumbar pars interarticularis: the development of a new MRI classification system. Spine 2002;27:181-86

4. Morrison JL, Kaplan PA, Dussault RG, et al. Pedicle marrow signal intensity changes in the lumbar spine: a manifestation of facet degenerative joint disease. Skeletal Radiol 2000;29:703-07

5. Sairyo K, Katoh S, Takata Y, et al. MRI signal changes of the pedicle as an indicator for early diagnosis of spondylolysis in children and adolescents: a clinical and biomechanical study. Spine 2006;31:206-11

6. Modic MT, Steinberg PM, Ross JS, et al. Degenerative disk disease: assessment of changes in vertebral body marrow with MR imaging. Radiology 1988;166 193-99

7. Rahme R, Moussa R. The Modic vertebral endplate and marrow changes: pathologic significance and relation to low back pain and segmental instability of the lumbar spine. AJNR Am J Neuroradiol 2008;:838-42

8. Modic MT, Obuchowski NA, Ross JS, et al. Acute low back pain and radiculopathy: MR imaging findings and their prognostic role and effect on outcome. Radiology 2005;237:597-604

9. Sairyo K, Katoh S, Takata Y, et al. MRI signal changes of the pedicle as an indicator for early diagnosis of spondylolysis in children and adolescents. Spine 31:206-211

10. Arendt EA, Griffiths HJ. The use of MR imaging in the assessment and clinical management of stress reactions of bone in high-performance athletes. Clin Sports Med 1997;16:291-306

11. Lee JC, Malara FA, Wood T, et al. MRI of stress reaction of the distal humerus in elite tennis players. Am J Roentgenol 2006;187:901-04

12. Sormaala MJ, Niva MH, Kiuru MJ, et al. Bone stress injuries of the talus in military recruits. Bone 2006;39:199-204

13. Schweitzer ME, White LM. Does altered biomechanics cause marrow edema? Radiology 1996;198:851-53

14. Fredericson M, Bergman AG, Hoffman KL, et al. Tibial stress reaction in runners: correlation of clinical symptoms and scintigraphy with a new magnetic resonance imaging grading system. Am J Sports Med 1995;23:472-81

15. Mitra D, Cassar-Pullicino VN, McCall IW. Longitudinal study of vertebral type-1 end-plate changes on MR of the lumbar spine. Eur Radiol 2004;14:1574-81

16. Modic MT. Modic type 1 and type 2 changes. J Neurosurg Spine 2007;6:150-51

17. Vital JM, Gille O, Pointillart V, et al. Course of Modic 1 six months after lumbar posterior osteosynthesis. Spine 2003;28:715-21

18. Toyone $\mathrm{T}$, Takahashi $\mathrm{K}$, Kitahara $\mathrm{H}$, et al. Vertebral bone-marrow changes in degenerative lumbar disc disease: an MRI study of 74 patients with low back pain. J Bone Joint Surg Br 1994;76:757-64

19. Kuisma M, Karppinen J, Niinimaki J, et al. Modic changes in endplates of lumbar vertebral bodies: prevalence and association with low back and sciatic pain among middle-aged male workers. Spine 2007;32:1116-22

20. Albert HB, Manniche C. Modic changes following lumbar disc herniation. Eur Spine J 2007;16:977-82

21. Ross JS, Zepp R, Modic MT. The postoperative lumbar spine: enhanced MR evaluation of the intervertebral disk. AJNR Am J Neuroradiol 1996;17:323-31

22. Masaryk TJ, Boumphrey F, Modic MT, et al. The effects of chemonucleolysis demonstrated by magnetic resonance imaging. J Comput Assist Tomogr $1986 ; 10: 917-23$

23. Lang P, Chafetz N, Genant HK, et al. Lumbar spinal fusion: assessment of functional stability with magnetic resonance imaging. Spine 1990; 15:581-88

24. Buttermann GR, Heithoff KB, Ogilvie JW, et al. Vertebral body MRI related to lumbar fusion results. Eur Spine J 1997;6:115-20

25. Chataigner H, Onimus M, Polette A. Surgery for degenerative lumbar disc disease: should the black disc be grafted? Rev Chir Orthop Reparatrice Appar Mot 1998;84:583-89

26. Esposito P, Pinheiro-Franco JL, Froelich S, et al. Predictive value of MRI vertebral end-plate signal changes (Modic) on outcome of surgically treated degenerative disc disease: results of a cohort study including 60 patients. Neurochirurgie 2006;52:315-22

27. Chamay A, Tschantz P. Mechanical influences in bone remodeling. Experimental research on Wolff's law. J Biomech 1972;5:173-80

28. Carter D. Mechanical loading histories and cortical bone remodeling. Calcif Tissue Int 1984;36(Suppl 1):S19-24

29. Roub LW, Gumerman LW, Hanley EN, et al. Bone stress: a radionuclide imaging perspective. Radiology 1997; 132:431-38

30. Daffner RH, Pavlov H. Stress fractures: current concepts. AJR Am J Roentgenol 1992;159:245-52

31. Mink JH, Deutsch AL. Occult cartilage and bone injuries of the knee: detection, classification, and assessment with MR imaging. Radiology 1989;170(3 Pt 1):823-29

32. Jaworski ZF. Coupling of bone formation to bone resorption: a broader view. Calcif Tissue Int 1984;36:531-35

33. Lakadamyali H, Tarhan NC, Cakir B, et al. STIR sequence for depiction of degenerative changes in posterior stabilizing elements in patients with lower back pain. AJR Am J Roentgenol 2008;191:973-79

34. D'Aprile P, Tarantino A, Jinkins JR, et al. The value of fat saturation sequences and contrast medium administration in MRI of degenerative disease of the posterior/perispinal elements of the lumbosacral spine. Eur Radiol 2007; 17:523-31

35. Sakai, T, Sairyo K, Mima S, et al. Significance of magnetic resonance imaging spinal change in the pedicle in the management of pediatric lumar spondylolysis. Spine 2010;34:E641-45 